\title{
On-Surface Synthesis of Chlorinated Narrow Graphene Nanoribbon Organometallic Hybrids
}

Rafal Zuzak, Pedro Brandimarte, Piotr Olszowski, Irena Izydorczyk, Marios Markoulides, Bartosz Such, Marek Kolmer, Marek Szymonski, Aran Garcia-Lekue, Daniel Sánchez-Portal, André Gourdon, and Szymon Godlewski*

Cite This: J. Phys. Chem. Lett. 2020, 11, 10290-10297

Read Online

ACCESS | 네 Metrics \& More | 回 Article Recommendations | sl Supporting Information

ABSTRACT: Graphene nanoribbons (GNRs) and their derivatives attract growing attention due to their excellent electronic and magnetic properties as well as the finetuning of such properties that can be obtained by heteroatom substitution and/or edge morphology modification. Here, we introduce graphene nanoribbon derivativesorganometallic hybrids with gold atoms incorporated between the carbon skeleton and side $\mathrm{Cl}$ atoms. We show that narrow chlorinated 5-AGNROHs (armchair graphene nanoribbon organometallic hybrids) can be fabricated by on-surface polymerization with omission of the cyclodehydrogenation reaction by a proper choice of tailored molecular precursors. Finally, we describe a route to exchange chlorine atoms connected through gold atoms to the carbon skeleton by hydrogen atom treatment. This is achieved directly on the surface, resulting in perfect unsubstituted hydrogen-terminated GNRs. This will be beneficial in the molecule on-surface processing when the preparation of final unsubstituted hydrocarbon structure is desired.

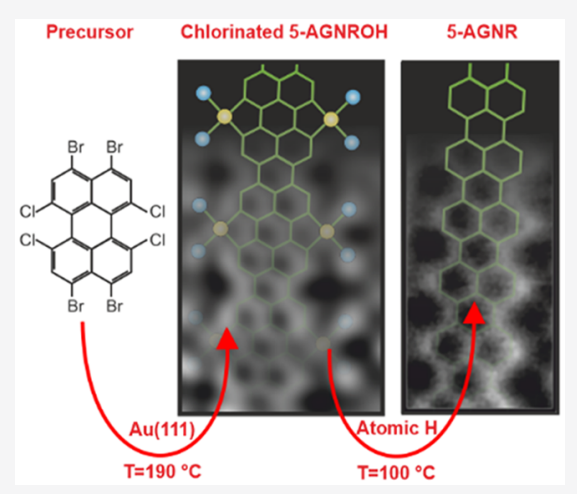

G raphene nanoribbons (GNRs) and nanographenes are regarded as promising candidates for the next generation of nano- and optoelectronic applications. ${ }^{1-6}$ While this new class of materials retains outstanding transport properties characteristic of graphene (e.g., high mobility of charge carriers), the lateral confinement of armchair graphene nanoribbons (AGNRs) opens a band gap. This is crucial in overcoming the most important obstacle of graphene-based transistors to applications in digital electronics and highfrequency circuitry, ${ }^{7}$ that is, the inability to completely turn them off at room temperature. On one hand, GNR electronic and magnetic properties strongly depend on the edge morphology; for example, the zigzag edge structure is predicted to result in spin-polarized edge states with potential applications in spintronic devices. ${ }^{8,9}$ On the other hand, armchair GNRs are characterized by a band gap dependent on their width, ${ }^{10-13}$ and the researcher's attention is also drawn to topological modifications by inclusion of nonhexagonal rings. ${ }^{14}$ The electronic and magnetic properties of GNRs could also be influenced and tuned by intentional substitutional doping. ${ }^{15-26}$

The preparation of these well-defined narrow GNRs has been made possible by advances in on-surface synthesis in ultrahigh-vacuum conditions. Most often the on-surface synthesis of GNRs proceeds in two sequential steps: (a) the halogen-equipped molecular precursors are activated thermally by carbon-halogen bond cleavage followed by Ullmann coupling to form polymers ${ }^{27,28}$ and (b) at higher temperature the oligomers are transformed into GNR by surface-assisted cyclodehydrogenation. $^{29}$ However, a single-step synthesis avoiding the high-temperature on-surface cyclodehydrogenation $^{30-32}$ would be instrumental for the fabrication of nanostructured ribbons which cannot tolerate high-temperature treatment. Among different molecular nanostructures created through surface-assisted synthesis, organometallic architectures attract in recent years growing attention as a promising class of materials. The family of fused, electronically delocalized organometallic polymers has been widely studied in solution. They potentially allow rational fine-tuning of the electronic, electrochemical, optical, and magnetic properties by controlling both the chemical structures of the organic ligands and the metallic centers. For instance, Osuka and collaborators have developed long molecular ladders comprising up to 24 porphyrin subunits. ${ }^{33,34}$ However, the stepwise synthesis of such systems in solution is complex and time-consuming, and thus it is tempting to explore on-surface polymerization as a rapid and efficient route to fused organometallic complexes. $^{35-51}$

Here we demonstrate the on-surface synthesis of specific GNR derivatives, that is, stable narrow chlorinated graphene

Received: October 15, 2020

Accepted: November 16, 2020

Published: November 23, 2020 


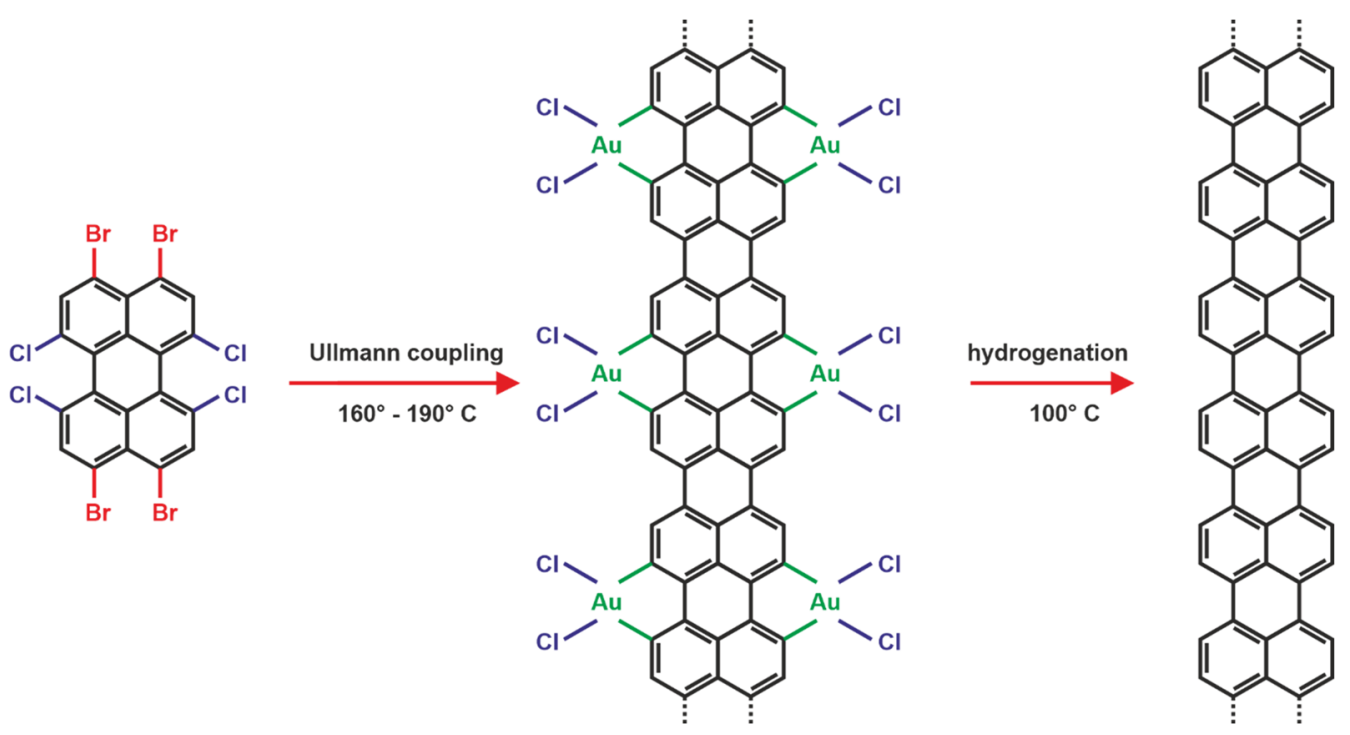

Figure 1. Synthetic road for on-surface fabrication of chlorinated 5-AGNROHs and bare 5-AGNR on the Au(111) surface.

nanoribbon organometallic hybrids (GNROHs). They are synthesized without the cyclodehydrogenation reaction by onsurface polymerization associated with organometallic coupling of specially designed tailored molecular precursors. In order to fabricate GNROHs in a single Ullmann coupling step, 1,6,7,12tetrachloro-3,4,9,10-tetrabromoperylene-equipped with four $\mathrm{Br}$ and four $\mathrm{Cl}$ side substituents-was synthesized. ${ }^{52}$ After onsurface activation by $\mathrm{C}-\mathrm{Br}$ bond cleavage, the precursor allowed for simultaneous $\mathrm{C}-\mathrm{C}$ bond formation and aromatic ring closure associated with complex formation with $\mathrm{Au}$ incorporated between two pairs of $\mathrm{C}-\mathrm{Cl}$ bonds. This leads to chlorinated 5-AGNROHs synthesized directly on the $\mathrm{Au}(111)$ surface (see Figure 1) with 4-fold-coordinated $\mathrm{Au}$ atoms, similarly as in gold-organic hybrids reported by Zhang et al. ${ }^{44}$ and Liu et al. ${ }^{53}$ Our approach creates also perspectives for the synthesis of GNRs and their derivatives directly on semiconducting and insulating substrates, where the controlled surface-assisted cyclodehydrogenation reaction does not occur. $^{54,55}$ Moreover, following our recent report, ${ }^{30}$ we demonstrate that by exposing the fabricated GNROHs to atomic hydrogen, we induce reduction of the gold complex to yield atomically perfect unsubstituted 5-AGNRs (Figure 1).

The precursor monomers synthesized following Zagryanski et $\mathrm{al}^{52}$ were sublimed on the $\mathrm{Au}(111)$ surface kept at room temperature, where they self-assemble into islands (see Figure S1 of the Supporting Information). Further slow annealing up to $160-190{ }^{\circ} \mathrm{C}$ induces $\mathrm{C}-\mathrm{Br}$ bond cleavage that activates the molecular building blocks. Subsequently, the activated molecules undergo polymerization reaction, and each two neighboring precursors are fused, forming two new covalent bonds, while within the same step the $\mathrm{C}-\mathrm{Cl}$ bonds transform into organometallic hybrids with $\mathrm{Au}$ atoms inserted between carbon skeleton and side $\mathrm{Cl}$ atoms (Figure 1). Such a transformation resembles the process observed for $\mathrm{Br} 4$ PTCDA precursors by Liu et al. ${ }^{50}$ The chlorinated 5AGNROHs are formed at an annealing temperature significantly lower compared to standard procedures involving the cyclodehydrogenation process. ${ }^{29}$ Figure $2 \mathrm{a}, \mathrm{c}$ shows a scanning tunneling microscopy (STM) overview image of the synthesized structures. There are two particular assemblies that could be discerned, namely, the islands comprised of linearly extended objects and dotted structures characterized by a
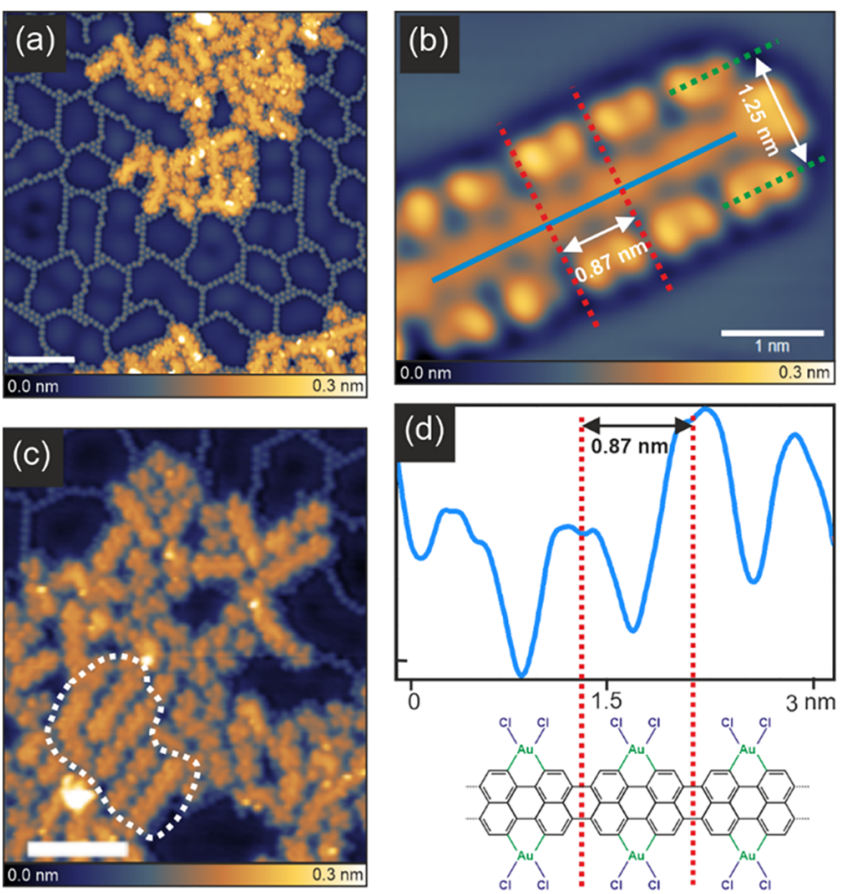

Figure 2. On-surface synthesized chlorinated 5-AGNROHs: (a) filled state STM overview with clearly visible ribbons and the quasihexagonal pattern formed by dotted lines of $\mathrm{Br}$ atoms indicated by a white arrow; (b) high-resolution STM image with intramolecular contrast showing a chlorinated 5-AGNROH; (c) magnified STM image of the short ribbon hybrid assemblies, where the dashed white contour marks longer ribbon hybrids with their tendency to parallel arrangement; (d) cross section along the $\mathrm{Cl}$ equipped 5-AGNROH measured along the blue line in (b) and a schematic structural model. Scanning parameters: $-1 \mathrm{~V}, 100 \mathrm{pA}$; scale bar $(\mathrm{a}, \mathrm{c}) 5 \mathrm{~nm}$.

quasi-hexagonal ordering. We attribute the first to the islandtype assemblies of chlorinated 5-AGNROHs. To further support the assignment, we analyze in detail the highresolution STM topography displayed in Figure $2 \mathrm{~b}$. The STM image contains the linear backbone complemented by large lobes located sideways. The linear structure could be assigned to the aromatic 5-AGNR core of the hybrid. The 
measured spacing between neighboring tailored building block units of $0.87 \pm 0.03 \mathrm{~nm}$ matches perfectly the anticipated periodicity of the aromatic 5-AGNR structure as indicated in Figure $2 \mathrm{~b}, \mathrm{~d}$. The large side lobes could be attributed to the presence of $\mathrm{Cl}$ side substituents. The spacing of these lobes across the molecular structure reaching $1.25 \pm 0.03 \mathrm{~nm}$ (see Figure $2 \mathrm{~b}$ ) is too large for a direct $\mathrm{C}-\mathrm{Cl}$ connection. This suggests incorporation of $\mathrm{Au}$ adatoms between the carbon skeleton and side $\mathrm{Cl}$ atoms, which would lead to on-surface synthesis of the chlorinated complex. Altogether this suggests attachment of $\mathrm{Cl}$ atoms to the aromatic skeleton through 4fold-coordinated Au adatoms after annealing to $160-190{ }^{\circ} \mathrm{C}$. In the STM image visualized in Figure $2 b$, each lobe corresponds to one of two neighboring $\mathrm{Cl}$ side atoms.

The dotted structures surrounding the islands with chlorinated 5-AGNROHs are formed by $\mathrm{Br}$, which gathers on the surface after $\mathrm{C}-\mathrm{Br}$ bond cleavage and precursor activation, similarly as it was recently reported by Zuzak et al. ${ }^{30}$ and Sun et al. ${ }^{32}$ We emphasize at this point that the GNROHs are formed at temperature below $200{ }^{\circ} \mathrm{C}$, which is lower than the desorption temperature of bromine atoms. ${ }^{31,56}$ This results in the clearly discernible appearance of $\mathrm{Br}$ on the surface as the reaction byproduct. Moreover, as coupling reactions yield four bromine atoms per building block, a large number of bromine atoms are released and form the quasi-hexagonal assemblies identical with the structures reported by Gottardi ${ }^{57}$ and also recently by Sun et al. ${ }^{32}$ Although the synthesized chlorinated 5AGNROHs gather into islands, we note that unsubstituted 5AGNRs do not exhibit at all any tendency to aggregate and stay well separated in the absence of bromine. ${ }^{31,88}$ Contrarily, similar self-assembly into islands of 5-AGNRs surrounded by bromine has recently also been reported. ${ }^{32}$ Furthermore, it is interesting to note that the assembly of the chlorinated 5AGNROHs is dependent on their length. In Figure $2 c$, we mark the longer ribbons, which evidently assemble parallel to each other. The shorter molecular species containing no more than 3 molecular units do not exhibit such tendency of higher order self-assembly as can be seen in the surrounding areas. These findings suggest that the ordering of longer ribbon assemblies emerges from the presence of chlorinated long edges, whose role in the intermolecular coupling is enhanced when the length of the ribbon increases. To analyze the hypothesis, we prepared a sample with reduced density of precursors, which may enable the creation of longer GNR hybrids, for example, due to a decreased amount of residual bromine, which is expected to influence the precursor mobility. This is documented in Figure 3a,b showing the coexistence of chlorinated GNROHs with increased length and assembled into ordered islands and less ordered groups of shorter ones. It is interesting to note that the amount of surrounding $\mathrm{Br}$ structures is greatly reduced due to the diminished density of tailored building blocks. From the high-resolution images shown in Figure 3c,d, it is evident that the long GNROHs tend to gather parallel to each other.

The STM images clearly reveal that if the number of sublimed molecules is reduced, the $\mathrm{Au}(111)$ surface herringbone reconstruction is absent in the surface region covered by the $\mathrm{Br}$ structures (see Figure $2 \mathrm{a}, \mathrm{c}$ ) and appear only in the clean area in-between the self-assembled pattern, as shown in Figure 3a,b. It is evident that $\mathrm{Br}$ atoms present on the surface may also significantly influence the GNR mobility. Consequently, the removal of $\mathrm{Br}$ atoms from the surface could be beneficial. One way to remove $\mathrm{Br}$, usually applied when
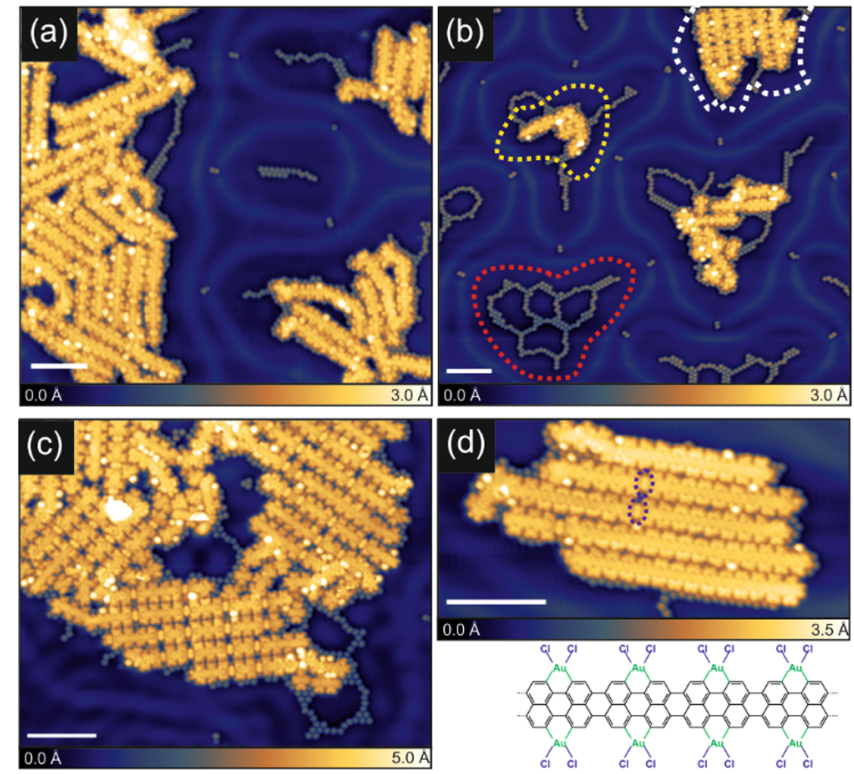

Figure 3. On-surface synthesized chlorinated 5-AGNROHs: (a) filled state STM overview with well visible islands comprising GNROHs; (b) filled state STM image showing smaller GNROH assemblies (dashed yellow contour) surrounded by bromine, quasi-hexagonal ordering of bromine (dashed red contour), ordered GNROH island (dashed white contour), and a clearly visible herringbone surface reconstruction around $\mathrm{Br}$ and $\mathrm{GNROH}$ assemblies; (c, d) highresolution filled state STM images visualizing parallel ordering of neighboring GNRs within molecular islands containing longer ribbons. Scanning parameters: $(\mathrm{a}-\mathrm{c})-1 \mathrm{~V}, 150 \mathrm{pA},(\mathrm{d})-0.5 \mathrm{~V}$, $100 \mathrm{pA}$; scale bar: $5 \mathrm{~nm}$.

GNRs are formed by cyclodehydrogenation, is thermal desorption. It is reported that the process takes place at temperatures above $250{ }^{\circ} \mathrm{C},{ }^{56}$ higher than the chlorine ${ }^{59}$ or fluorine $^{60}$ equipped aromatic platforms can survive. Therefore, to analyze the role of surface $\mathrm{Br}$ atoms on the molecule behavior, we have applied an alternative procedure that has been proposed for postpolymerization Br removal. ${ }^{61,62}$ This is done by exposure of the sample to molecular $\mathrm{H}_{2}$ flux. We kept the sample heated to $180{ }^{\circ} \mathrm{C}$ with the $\mathrm{H}_{2}$ partial pressure at 5 $\times 10^{-7}$ mbar. The heating temperature was selected to ensure efficient $\mathrm{Br}$ removal while maintaining the chlorinated GNROH intact. Low annealing temperatures did not result in surface cleaning from $\mathrm{Br}$ structures; for example, annealing at $150{ }^{\circ} \mathrm{C}$ for $2 \mathrm{~h}$ had no impact on the observed $\mathrm{Br}$ structures. The experiments showed that almost complete $\mathrm{Br}$ removal from the surface requires at least $2.5 \mathrm{~h}$ treatment at $180{ }^{\circ} \mathrm{C}$ as shown in the STM image in Figure 4. The image shows that

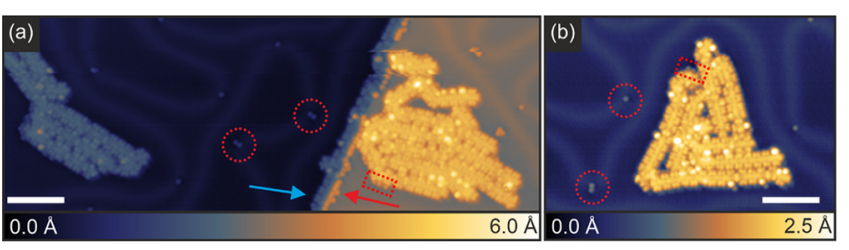

Figure 4. (a, b) Filled state STM images of chlorinated 5-AGNROHs after postsynthesis surface cleaning using $2.5 \mathrm{~h}$ molecular $\mathrm{H}_{2}$ treatment at $180{ }^{\circ} \mathrm{C}$. The red dashed circles, red arrow, and red dashed rectangle indicate residual bromine contamination; the blue arrow points to the molecules immobilized at a terrace step. Scanning parameters: $-1 \mathrm{~V}, 100 \mathrm{pA}$; scale bar: $5 \mathrm{~nm}$. 
the $\mathrm{Br}$ structures disappeared, with some $\mathrm{Br}$ adatoms left, especially at the terrace edges, in the vicinity of surface reconstruction corners and surrounding the GNROHs. They are indicated in Figure 4 by the red dotted circles, red arrow, and red dashed rectangle. We can also expect that some $\mathrm{Br}$ atoms might still be present between the parallel oriented 5AGNROHs. The surface herringbone pattern is restored after $\mathrm{Br}$ extraction in the area surrounding the GNROH islands, whereas it is clear that underneath the GNROHs it does not appear. Thus, our observation points out to either noticeable interaction between the GNROHs and the surface, most likely arising from the edge functionalization with $\mathrm{Cl}$ and formation of organometallic complex, or the still important role of the $\mathrm{Br}$ adatoms buried within the assemblies of the 5-AGNROHs.

As described above, the application of molecular hydrogen is not capable of complete residual bromine removal. Alternatively, bromine atoms may be removed from the surface either by deposition of silicon atoms ${ }^{32}$ or by atomic hydrogen dosing, ${ }^{30,63}$ the application of which has recently been introduced in on-surface synthesis approach for intermolecular fusion. ${ }^{64}$ It is likely that application of atomic hydrogen shall be much more efficient in removing residual halogen atoms; ${ }^{30}$ however, it may also modify the generated gold complexes. Indeed, in the following we show that the application of atomic hydrogen to GNROHs leads to organometallic state quenching and passivation of the carbon skeleton providing perfect 5AGNRs. In Figure 5a, a typical overview image of the sample
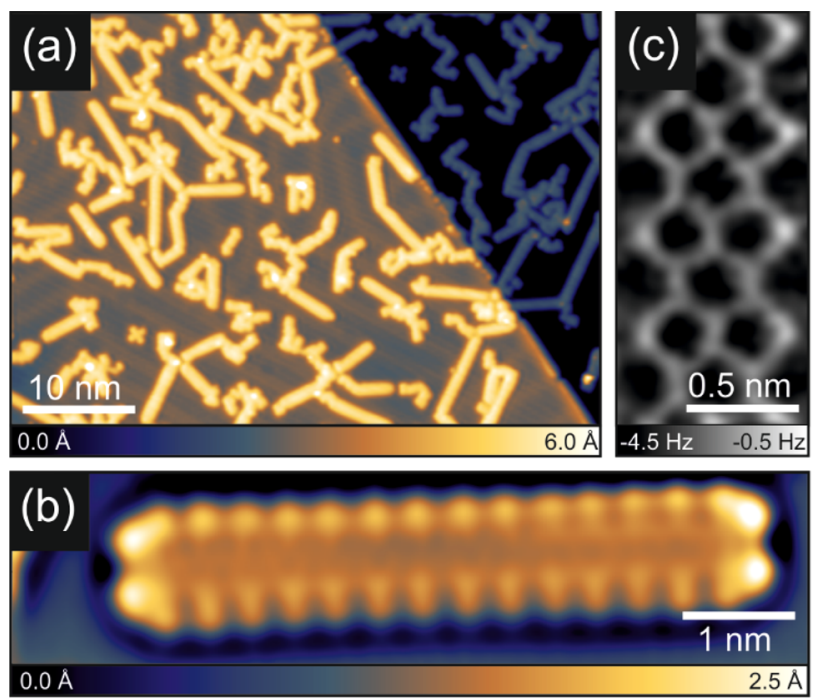

Figure 5. Unsubstituted GNRs obtained by the exchange of $\mathrm{Cl}$ atoms by $\mathrm{H}$ species performed directly on the $\mathrm{Au}(111)$ surface by the application of atomic $\mathrm{H}$ at $100{ }^{\circ} \mathrm{C}$ : (a) filled state overview STM image showing separated 5-AGNRs; (b) high-resolution STM image of the 5-AGNR comprising seven molecular precursors; (c) Laplacefiltered frequency shift nc-AFM image of the 5-AGNR with well visible aromatic skeleton and hydrogen-terminated edges. Scanning parameters: $(\mathrm{a}, \mathrm{b})-1 \mathrm{~V}, 200 \mathrm{pA}$.

exposed to the atomic hydrogen source is presented. It is apparent that the application of the atomic hydrogen treatment strongly influences the GNROHs. During the process the sample is kept at elevated temperature of $100{ }^{\circ} \mathrm{C}$ with a partial pressure of $1 \times 10^{-7} \mathrm{mbar}$ for $15 \mathrm{~min}$. STM images show that previously generated GNROHs become separated, and their appearance closely resembles the unsubstituted 5-AGNRs observed by Kimouche et al., ${ }^{58,66}$ as can be also observed in a high-resolution image of the GNR containing seven molecular precursors in Figure $5 \mathrm{~b}$. Remarkably, the herringbone surface pattern is no longer affected by the presence of the GNRs, strongly indicating that the $\mathrm{Cl}$ side atoms and the previously residual $\mathrm{Br}$ adatoms are successfully removed by the applied procedure. This supports the efficiency of halogen atom removal from noble metal surfaces by atomic hydrogen ${ }^{30,64}$ also in cases where the halogen atom is a part of an organometallic gold complex.

However, we note here that the analysis of the molecular structure solely based on STM visualization might be misleading due the complicated influence of topography and electronic structure on the resulting image. To perform a doubtless identification of the product of the reaction, we apply the noncontact atomic force microscopy (nc-AFM) technique with a $\mathrm{CO}$ functionalized tip. It has been shown that such functionalization allows not only for the detailed imaging of the internal structure of organic species ${ }^{65,66}$ but also for the precise analysis of the molecular edges. ${ }^{67,68}$ The image shown in Figure $5 \mathrm{c}$ indicates the presence of the 5-AGNR aromatic core without $\mathrm{Cl}$ atoms but with hydrogen-terminated edges. This proves that the applied procedure leads to the pristine 5AGNRs containing $\mathrm{sp}^{2}$-hybridized $\mathrm{C}$ atoms without superhydrogenated edges. ${ }^{64}$ In contrast, an edge with radical character or saturated with two $\mathrm{H}$ atoms would lead to a darker or brighter appearance, respectively. ${ }^{69,70}$ Therefore, we conclude that the exposure to atomic $\mathrm{H}$ source not only cleans the surface completely from $\mathrm{Br}$ adatoms but also leads to the full quenching of the organometallic complex leading to perfect unsubstituted 5-AGNRs.

The atomic hydrogen treatment gives also a chance for unambiguous identification of the chlorinated 5-AGNROHs. Whereas there are reports showing successful measurements with $\mathrm{CO}$ functionalized tips in the presence of $\mathrm{Br}$ on $\operatorname{Ag}(111),{ }^{71}$ we did not manage to functionalize the nc-AFM tip with a $\mathrm{CO}$ molecule on $\mathrm{Au}(111)$ containing $\mathrm{Br}$ contamination $^{30}$ due to noticeable interaction between $\mathrm{Br}$ adatoms with sublimed $\mathrm{CO}$ molecules. To overcome these difficulties, we can apply a shorter lasting exposure of the sample containing 5-AGNROHs to atomic hydrogen, which allows to effectively get rid of the remaining $\mathrm{Br}$ adatoms ${ }^{30}$ and removes only a part of $\mathrm{Cl}$ side substituents within AGNROHs, transforming them into 5-AGNR/5-AGNROH heterojunctions. This is documented in Figure $6 \mathrm{a}, \mathrm{b}$, which allows for the doubtless identification of the 5AGNR/5AGNROH junctions and for the precise description of their atomic composition based on their nc-AFM appearance. The nc-AFM image in Figure 6a corroborates presence of 4-fold coordinated $\mathrm{Au}$ atoms located between the carbon-based GNR skeleton and side $\mathrm{Cl}$ substituents as schematically drawn for clarity in Figure 6b. An additional STM image of partially dechlorinated AGNROHs can be found in Figure S2.

To support the existence and stability of chlorinated 5AGNROHs on $\mathrm{Au}(111)$, we have performed density functional theory (DFT)-based calculations of their structural arrangement (see the Supporting Information for calculation details). ${ }^{72}$ The optimized structure of the chlorinated 5AGNROH complex on $\mathrm{Au}(111)$ is shown in Figure 6c,d. The calculations indicate a slightly bent geometry of the chlorinated AGNROH with both sides comprising $\mathrm{Cl}$ atoms facing toward the $\mathrm{Au}(111)$ surface. Between $\mathrm{Cl}$ and $\mathrm{C}$ atoms, 4-fold-coordinated $\mathrm{Au}$ atoms are incorporated forming a complex, in a similar way as reported by Liu et al. ${ }^{57}$ The 

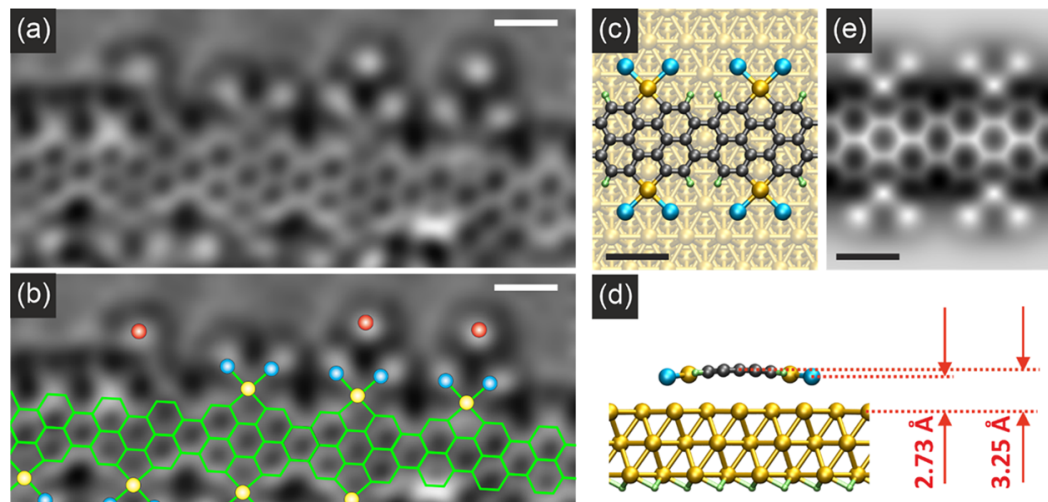

Figure 6. (a, b) Partially hydrogenated chlorine-doped 5-AGNROH, Laplace-filtered frequency shift nc-AFM image of the 5-AGNR/5-AGNROH with well visible aromatic skeleton and organometallic moieties; the molecular nanostructure is still surrounded by residual $\mathrm{Br}$ atoms, as inferred from comparison with STM images (see Figure S3). (c, d) Optimized structure of chlorinated 5-AGNROH. (e) Simulated nc-AFM image of chlorinated 5-AGNROH. Scale bar: $0.5 \mathrm{~nm}$. Color coding: yellow $=\mathrm{Au}$ atoms, blue $=\mathrm{Cl}$ atoms, red $=\mathrm{Br}$ adatoms, and green $=\mathrm{H}$ atoms.

calculated height over the gold surface of the highest lying central carbon atoms reaches $\sim 3.25 \AA$, which is slightly-in the order of $0.1 \AA$-closer to the Au plane than in the case of unsubstituted, pristine 5-AGNRs. Chlorine atoms are located $\sim 2.73 \AA$ above the Au plane, noticeably closer than $\mathrm{C}$ atoms, as indicated in Figure $6 \mathrm{~d}$. On the basis of the obtained geometrical architecture, we have performed nc-AFM image simulations following the approach introduced by Hapala et al. $^{73,74}$ The resulting simulated nc-AFM image presented in Figure $6 \mathrm{e}$ matches well with the experimentally captured organometallic units in Figure $6 \mathrm{a}, \mathrm{b}$, providing confirmation of the successful fabrication of the chlorinated 5-AGNROHs.

In conclusion, we have fabricated a chlorinated organometallic 5-AGNR complex on the $\mathrm{Au}(111)$ surface, which could be regarded as representatives of a special class of compounds: GNR organometallic derivatives. The nanostructures have been generated in a single-step procedure involving only the on-surface polymerization process accompanied by the formation of the organometallic state with omission of the cyclodehydrogenation. Consequently, the GNROHs are formed at significantly lower temperature than following a standard GNR fabrication procedure, which altogether enables the incorporation of $\mathrm{Au}$ atoms between two $\mathrm{C}-\mathrm{Cl}$ bonds and the synthesis of organometallic complex. Our approach opens up new prospects for the fine-tuning of the electronic properties of GNRs and their derivatives as well as for the enhancement of their processability and the fabrication of nanocomposites. The on-surface synthesis is achieved by application of specially designed molecular precursors, each equipped with four $\mathrm{Br}$ and four $\mathrm{Cl}$ atoms. This allows for simultaneous $\mathrm{C}-\mathrm{C}$ bond formation and aromatic ring closure. Such a procedure is expected to be highly beneficial when applied on other surfaces, which do not promote the metal catalyzed on-surface cyclodehydrogenation, and gives perspectives for direct fabrication of GNRs on nonmetallic substrates by homolytic cleavage of $\mathrm{C}-\mathrm{X}$ bonds. ${ }^{75}$ Finally, it is demonstrated that chlorinated GNROHs could be transformed into unsubstituted, H-terminated perfect GNRs by the onsurface side atom exchange using an atomic $\mathrm{H}$ source at elevated temperature. This is expected to be beneficial in the synthesis of a wide range of molecular moieties giving a powerful tool to end up with purely hydrocarbon structures or heterojunctions.

\section{ASSOCIATED CONTENT}

\section{Supporting Information}

The Supporting Information is available free of charge at https://pubs.acs.org/doi/10.1021/acs.jpclett.0c03134.

Experimental methods, computational details and additional STM data, calculated electronic structure of AGNROHs (PDF)

\section{AUTHOR INFORMATION}

\section{Corresponding Author}

Szymon Godlewski - Centre for Nanometer-Scale Science and Advanced Materials, NANOSAM, Faculty of Physics, Astronomy and Applied Computer Science, Jagiellonian University, PL 30-348 Krakow, Poland; orcid.org/00000002-8515-1566; Email: szymon.godlewski@uj.edu.pl

\section{Authors}

Rafal Zuzak - Centre for Nanometer-Scale Science and Advanced Materials, NANOSAM, Faculty of Physics, Astronomy and Applied Computer Science, Jagiellonian University, PL 30-348 Krakow, Poland; (1) orcid.org/00000001-6617-591X

Pedro Brandimarte - Donostia International Physics Center, E-20018 Donostia-San Sebastián, Spain; (1) orcid.org/00000002-8762-5876

Piotr Olszowski - Centre for Nanometer-Scale Science and Advanced Materials, NANOSAM, Faculty of Physics, Astronomy and Applied Computer Science, Jagiellonian University, PL 30-348 Krakow, Poland

Irena Izydorczyk - Centre for Nanometer-Scale Science and Advanced Materials, NANOSAM, Faculty of Physics, Astronomy and Applied Computer Science, Jagiellonian University, PL 30-348 Krakow, Poland; (1) orcid.org/00000003-1561-119X

Marios Markoulides - CEMES-CNRS (UPR 8011), 31055 Cedex 4 Toulouse, France

Bartosz Such - Centre for Nanometer-Scale Science and Advanced Materials, NANOSAM, Faculty of Physics, Astronomy and Applied Computer Science, Jagiellonian University, PL 30-348 Krakow, Poland; (1) orcid.org/00000001-9349-8247

Marek Kolmer - Centre for Nanometer-Scale Science and Advanced Materials, NANOSAM, Faculty of Physics, 
Astronomy and Applied Computer Science, Jagiellonian University, PL 30-348 Krakow, Poland; (orcid.org/00000002-6786-9697

Marek Szymonski - Centre for Nanometer-Scale Science and Advanced Materials, NANOSAM, Faculty of Physics, Astronomy and Applied Computer Science, Jagiellonian University, PL 30-348 Krakow, Poland

Aran Garcia-Lekue - Donostia International Physics Center, E-20018 Donostia-San Sebastián, Spain; IKERBASQUE, Basque Foundation for Science, E-48013 Bilbao, Spain; (1) orcid.org/0000-0001-5556-0898

Daniel Sánchez-Portal - Donostia International Physics Center, E-20018 Donostia-San Sebastián, Spain; Centro de Física de Materiales (CSIC-UPV/EHU), E-20018 DonostiaSan Sebastián, Spain

André Gourdon - CEMES-CNRS (UPR 8011), 31055 Cedex 4 Toulouse, France; 이이이.org/0000-0002-03701019

Complete contact information is available at: https://pubs.acs.org/10.1021/acs.jpclett.0c03134

\section{Notes}

The authors declare no competing financial interest.

\section{ACKNOWLEDGMENTS}

This work was supported by the National Science Center, Poland (2019/35/B/ST5/02666), and the EU Project PAMS (610446). A.G.L., D.S.P., and P.B. acknowledge the Spanish Agencia Estatal de Investigación (Grants MAT2016-78293C6-4-R, PID2019-107338RB-C66, and FIS2017-83780-P), Dep. Educación of the Basque Government and UPV/EHU (Grant IT-756-13), and the European Union (EU) through Horizon 2020 (FET-Open project SPRING Grant 863098) for support.

\section{REFERENCES}

(1) Li, X.; Wang, X.; Zhang, L.; Lee, S.; Dai, H. Chemically Derived, Ultrasmooth Graphene Nanoribbon Semiconductors. Science 2008, 319, 1229-1232.

(2) Kim, W. Y.; Kim, K. S. Prediction of Very Large Values of Magnetoresistance in a Graphene Nanoribbon Device. Nat. Nanotechnol. 2008, 3, 408.

(3) Han, M. Y.; Özyilmaz, B.; Zhang, Y.; Kim, P. Energy Band-Gap Engineering of Graphene Nanoribbons. Phys. Rev. Lett. 2007, 98, 206805.

(4) Son, Y.-W.; Cohen, M. L.; Louie, S. G. Energy Gaps in Graphene Nanoribbons. Phys. Rev. Lett. 2006, 97, 216803.

(5) Bennett, P. B.; Pedramrazi, Z.; Madani, A.; Chen, Y.-C.; de Oteyza, D. G.; Chen, C.; Fischer, F. R.; Crommie, M. F.; Bokor, J. Bottom-Up Graphene Nanoribbon Field-Effect Transistors. Appl. Phys. Lett. 2013, 103, 253114.

(6) Linas, J. P.; Fairbrother, A.; Barin, G. B.; Shi, W.; Lee, K.; Wu, S.; Choi, B. Y.; Braganza, R.; Lear, J.; Kau, N.; Choi, W.; Chen, C.; Pedramrazi, Z.; Dumslaff, T.; Narita, A.; Feng, X.; Müllen, K.; Fischer, F.; Zettl, A.; Ruffieux, P.; Yablonovitch, E.; Crommie, M. F.; Fasel, R.; Bokor, J. Short-Channel Field Effect Transistors with 9-Atom and 13Atom Wide Graphene Nanoribbons. Nat. Commun. 2017, 8, 633.

(7) Schwierz, F. Graphene Transistors. Nat. Nanotechnol. 2010, 5, 487-496.

(8) Son, Y.-W.; Cohen, M. L.; Louie, S. G. Half-Metallic Graphene Nanoribbons. Nature 2006, 444, 347-349.

(9) Ruffieux, P.; Wang, S.; Yang, B.; Sánchez-Sánchez, C.; Liu, J.; Dienel, T.; Talirz, L.; Shinde, P.; Pignedoli, C. A.; Passerone, D.; Dumslaff, T.; Feng, X.; Müllen, K.; Fasel, R. On-Surface Synthesis of
Graphene Nanoribbons with Zigzag Edge Topology. Nature 2016, 531, 489-493.

(10) Barone, V.; Hod, O.; Scuseria, G. E. Electronic Structure and Stability of Semiconducting Graphene Nanoribbons. Nano Lett. 2006, 6, 2748-2754.

(11) Yang, L.; Park, C.-H.; Son, Y.-W.; Cohen, M. L.; Louie, S. G. Quasiparticle Energies and Band Gaps in Graphene Nanoribbons. Phys. Rev. Lett. 2007, 99, 186801.

(12) Chen, Y.-C.; de Oteyza, D. G.; Pedramrazi, Z.; Chen, C.; Fischer, F. R.; Crommie, M. F. Tuning the Band Gap of Graphene Nanoribbons Synthesized from Molecular Precursors. ACS Nano 2013, 7, 6123-6128.

(13) Merino-Díez, N.; Garcia-Lekue, A.; Carbonell-Sanromà, E.; Li, J.; Corso, M.; Colazzo, L.; Sedona, F.; Sánchez-Portal, D.; Pascual, J. I.; De Oteyza, D. Width-Dependent Band Gap in Armchair Graphene Nanoribbons Reveals Fermi Level Pinning on $\mathrm{Au}(111)$. ACS Nano 2017, 11, 11661-11668.

(14) Rizzo, D. J.; Veber, G.; Jiang, J.; McRudy, R.; Cao, T.; Bronner, C.; Chen, T.; Louie, S. G.; Fischer, F. R.; Crommie, M. F. Inducing Metallicity in Graphene Nanoribbons via Zero-Mode Superlattices. Science 2020, 369, 1597-1603.

(15) Talirz, L.; Ruffieux, P.; Fasel, R. On-Surface Synthesis of Atomically Precise Graphene Nanoribbons. Adv. Mater. 2016, 28, 6222-6231.

(16) Cloke, R. R.; Marangoni, T.; Nguyen, G. D.; Joshi, T.; Rizzo, D. J.; Bronner, C.; Cao, T.; Louie, S. G.; Crommie, M. F.; Fischer, F. R. Site-Specific Substitutional Boron Doping of Semiconducting Armchair Graphene Nanoribbons. J. Am. Chem. Soc. 2015, 137, 88728875.

(17) Carbonell-Sanromà, E.; Brandimarte, P.; Balog, R.; Corso, M.; Kawai, S.; Garcia-Lekue, A.; Saito, S.; Yamaguchi, S.; Meyer, E.; Sánchez-Portal, D.; Pascual, J. I. Quantum Dots Embedded in Graphene Nanoribbons by Chemical Substitution. Nano Lett. 2017, $17,50-56$.

(18) Carbonell-Sanromà, E.; Hieulle, J.; Vilas-Varela, M.; Brandimarte, P.; Iraola, M.; Barragán, A.; Li, J.; Abadía, M.; Corso, M.; Sanchez-Portal, D.; Peña, D.; Pascual, J. I. Doping of Graphene Nanoribbons via Functional Group Edge Modification. ACS Nano 2017, 11, 7355-7361.

(19) Kawai, S.; Saito, S.; Osumi, S.; Yamaguchi, S.; Foster, A. S.; Spijker, P.; Meyer, E. Atomically Controlled Substitutional BoronDoping of Graphene Nanoribbons. Nat. Commun. 2015, 6, 8098.

(20) Vo, T. H.; Perera, U. G. E.; Shekhirev, M.; Pour, M. M.; Kunkel, D. A.; Lu, H.; Gruverman, A.; Sutter, E.; Cotlet, M.; Nykypanchuk, D.; Zahl, P.; Enders, A.; Sinitskii, A.; Sutter, P. Nitrogen-Doping Induced Self-Assembly of Graphene NanoribbonBased Two-Dimensional and Three-Dimensional Metamaterials. Nano Lett. 2015, 15, 5770-5777.

(21) Pedramrazi, Z.; Chen, C.; Zhao, F.; Cao, T.; Nguyen, G. D.; Omrani, A. A.; Tsai, H.-Z.; Cloke, R. R.; Marangoni, T.; Rizzo, D. J.; Joshi, T.; Bronner, C.; Choi, W.-W.; Fischer, F. R.; Louie, S. G.; Crommie, M. F. Concentration Dependence of Dopant Electronic Structure in Bottom-up Graphene Nanoribbons. Nano Lett. 2018, 18, $3550-3556$.

(22) Nguyen, G. D.; Toma, F. M.; Cao, T.; Pedramrazi, Z.; Chen, C.; Rizzo, D. J.; Joshi, T.; Bronner, C.; Chen, Y.-C.; Favaro, M.; Louie, S. G.; Fischer, F. R.; Crommie, M. F. Bottom-Up Synthesis of N = 13 Sulfur-Doped Graphene Nanoribbons. J. Phys. Chem. C 2016, 120, 2684-2687.

(23) Bronner, C.; Stremlau, S.; Gille, M.; Brauße, F.; Haase, A.; Hecht, S.; Tegeder, P. Aligning the Band Gap of Graphene Nanoribbons by Monomer Doping. Angew. Chem., Int. Ed. 2013, $52,4422-4425$.

(24) Clair, S.; de Oteyza, D. G. Controlling a Chemical Coupling Reaction on a Surface: Tools and Strategies for On-Surface Synthesis. Chem. Rev. 2019, 119, 4717-4776.

(25) Friedrich, N.; Brandimarte, P.; Li, J.; Saito, S.; Yamaguchi, S.; Pozo, I.; Pena, D.; Frederiksen, T.; Garcia-Lekue, A.; Sanchez-Portal, D.; Pascual, J. I. Magnetism of Topological Boundary States Induced 
by Boron Substitution in Graphene Magnetism of Topological Boundary States Induced by Boron Substitution in Graphene. Phys. Rev. Lett. 2020, 125, 146801.

(26) Carbonell-Sanroma, E.; Garcia-Lekue, A.; Corso, M.; Vasseur, G.; Brandimarte, P.; Lobo-Checa, J.; de Oteyza, D. G.; Li, J.; Kawai, S.; Saito, S.; Yamaguchi, S.; Ortega, E.; Sanchez-Portal, D.; Pascual, J. I. Electronic Properties of Substitutionally Boron-Doped Graphene Nanoribbons on a $\mathrm{Au}(111)$ Surface. J. Phys. Chem. C 2018, 122, 16092-16099.

(27) Grill, L.; Dyer, M.; Lafferentz, L.; Persson, M.; Peters, M. V.; Hecht, S. Nano-Architectures by Covalent Assembly of Molecular Building Blocks. Nat. Nanotechnol. 2007, 2, 687-691.

(28) Lafferentz, L.; Eberhardt, V.; Dri, C.; Africh, C.; Comelli, G.; Esch, F.; Hecht, S.; Grill, L. Controlling On-Surface Polymerization by Hierarchical and Substrate-Directed Growth. Nat. Chem. 2012, 4, 215-220.

(29) Cai, J.; Ruffieux, P.; Jaafar, R.; Bieri, M.; Braun, T.; Blankenburg, S.; Muoth, M.; Seitsonen, A. P.; Saleh, M.; Feng, X.; Müllen, K.; Fasel, R. Atomically Precise Bottom-Up Fabrication of Graphene Nanoribbons. Nature 2010, 466, 470-473.

(30) Zuzak, R.; Jančař́ik, A.; Gourdon, A.; Szymonski, M.; Godlewski, S. On-Surface Synthesis with Atomic Hydrogen. ACS Nano 2020, 14, 13316.

(31) Zhang, H.; Lin, H.; Sun, K.; Chen, L.; Zagranyarski, Y.; Aghdassi, N.; Duhm, S.; Li, Q.; Zhong, D.; Li, Y.; Müllen, K.; Fuchs, H.; Chi, L. On-Surface Synthesis of Rylene-Type Graphene Nanoribbons. J. Am. Chem. Soc. 2015, 137, 4022-4025.

(32) Sun, K.; Nishiuchi, T.; Sahara, K.; Kubo, T.; Foster, A. S.; Kawai, S. Low-Temperature Removal of Dissociated Bromine by Silicon Atoms for an On-Surface Ullmann Reaction. J. Phys. Chem. C 2020, 124, 19675-19680.

(33) Aratani, N.; Osuka, A.; Kim, Y. H.; Jeong, D. H.; Kim, D. Extremely Long, Discrete meso - meso-Coupled Porphyrin Arrays. Angew. Chem., Int. Ed. 2000, 39, 1458-1462.

(34) Ikeda, T.; Aratani, N.; Osuka, A. Synthesis of Extremely $\pi$ Extended Porphyrin Tapes from Hybrid meso-meso Linked Porphyrin Arrays: An Approach Towards the Conjugation Length. Chem. - Asian J. 2009, 4, 1248-1256.

(35) Liu, J.; Gao, Y.; Wang, T.; Xue, Q.; Hua, M.; Wang, Y.; Huang, L.; Lin, N. Collective Spin Manipulation in Antiferroelastic SpinCrossover Metallo-Supramolecular Chains. ACS Nano 2020, 14, 11283.

(36) Zhang, R.; Liu, J.; Gao, Y.; Hua, M.; Xia, B.; Knecht, P.; Papageorgiou, A.; Reichert, J.; Barth, J. V.; Huang, L.; Xu, H.; Lin, N. On-surface Synthesis of a Semiconducting 2D Metal-Organic Framework $\mathrm{Cu}_{3}\left(\mathrm{C}_{6} \mathrm{O}_{6}\right)$ Exhibiting Dispersive Electronic Bands. Angew. Chem., Int. Ed. 2020, 59, 2669-2673.

(37) Saywell, A.; Gren, W.; Franc, G.; Gourdon, A.; Bouju, X.; Grill, L. Manipulating the Conformation of Single Organometallic Chains on $\mathrm{Au}(111)$. J. Phys. Chem. C 2014, 118, 1719-1728.

(38) Piquero-Zulaica, I.; Sadeghi, A.; Kherelden, M.; Hua, M.; Liu, J.; Kuang, G.; Yan, L.; Ortega, J. E.; Abd El-Fattah, Z.; Azizi, B.; Lin, N.; Lobo-Checa, J. Electron Transmission Through Coordinating Atoms Embedded in Metal-Organic Nanoporous Networks. Phys. Rev. Lett. 2019, 123, 266805.

(39) Zhang, R.; Xia, B.; Xu, H.; Lin, N. Identifying Multi-Nuclear Organometallic Intermediates in On-Surface [2+2] Cycloaddition Reactions. Angew. Chem., Int. Ed. 2019, 58, 16485-16489.

(40) Yan, L.; Xia, B.; Zhang, Q.; Kuang, G.; Xu, H.; Liu, J.; Liu, P. N.; Lin, N. Stabilizing and Organizing Bi3Cu4 and $\mathrm{Bi} 7 \mathrm{Cu} 12$ Nanoclusters in Two-Dimensional Metal-Organic Networks. Angew. Chem., Int. Ed. 2018, 57, 4617-4621.

(41) Dong, L.; Gao, Z.; Lin, N. Self-Assembly of Metal-Organic Coordination Structures on Surfaces. Prog. Surf. Sci. 2016, 91, 101.

(42) Urgel, J.; Ecija, D.; Lyu, G.; Zhang, R.; Palma, C. A.; Auwärter, W.; Lin, N.; Barth, J. V. Quasicrystallinity Expressed in TwoDimensional Coordination Networks. Nat. Chem. 2016, 8, 657.

(43) Langner, A.; Tait, S. L.; Lin, N.; Chandrasekar, R.; Meded, V.; Fink, K.; Ruben, M.; Kern, K. Selective Coordination Read-out in
Surface-confined Metallo-supramolecular Systems. Angew. Chem., Int. Ed. 2012, 51, 4327-4331.

(44) Zhang, H.; Franke, J. H.; Zhong, D.; Li, Y.; Timmer, A.; Díaz Arado, O.; Mönig, H.; Wang, H.; Chi, L.; Wang, Z.; Müllen, K.; Fuchs, H. Surface Supported Gold-Organic Hybrids: On-Surface Synthesis and Surface Directed Orientation. Small 2014, 10, 13611368.

(45) Telychko, M.; Su, J.; Gallardo, A.; Gu, Y.; Mendieta-Moreno, J. I.; Qi, D.; Tadich, A.; Song, S.; Lyu, P.; Qiu, Z.; Fang, H.; Joo Koh, M.; Wu, J.; Jelínek, P.; Lu, J. Strain-Induced Isomerization in OneDimensional Metal-Organic Chains. Angew. Chem., Int. Ed. 2019, 58, 18591-18597.

(46) Li, Y.; Cheng, L.; Liu, C.; Liu, W.; Fan, Y.; Fan, X.; Zeng, Q. On-Surface Observation of the Formation of Organometallic Complex in a Supramolecular Network. Sci. Rep. 2015, 5, 10972.

(47) Zhang, C.; Sun, Q.; Kong, H.; Wang, L.; Tan, Q.; Xu, W. OnSurface Synthesis of Organometallic Complex via Metal-Alkene Interactions. Chem. Commun. 2014, 50, 15924-15927.

(48) Zhang, C.; Kazuma, E.; Kim, Y. Atomic-Scale Visualization of the Stepwise Metal-Mediated Dehalogenative Cycloaddition Reaction Pathways: Competition between Radicals and Organometallic Intermediates. Angew. Chem. 2019, 131, 17900-17908.

(49) Zhang, W.; Wang, T.; Han, D.; Huang, J.; Feng, L.; Ding, H.; $\mathrm{Hu}$, J.; Zeng, Z.; Xu, Q.; Zhu, J. Stepwise Synthesis of $\mathrm{N}-\mathrm{Ag}-\mathrm{N}$ and $\mathrm{C}-\mathrm{Ag}-\mathrm{C}$ Organometallic Structures on a $\mathrm{Ag}(111)$ Surface. J. Phys. Chem. C 2020, 124, 16415-16422.

(50) Fan, Q.; Wang, T.; Liu, L.; Zhao, J.; Zhu, J.; Gottfried, J. M. Tribromobenzene on $\mathrm{Cu}(111)$ : Temperature-Dependent Formation of Halogen-Bonded, Organometallic, and Covalent Nanostructures. J. Chem. Phys. 2015, 142, 101906.

(51) Urgel, J. I.; Hayashi, H.; Di Giovannantonio, M.; Pignedoli, C. A.; Mishra, S.; Deniz, O.; Yamashita, M.; Dienel, Y.; Ruffieux, P.; Yamada, H.; Fasel, R. On-Surface Synthesis of Heptacene Organometallic Complexes. J. Am. Chem. Soc. 2017, 139, 11658-11661.

(52) Zagranyarski, Y.; Chen, L.; Jansch, D.; Gessner, T.; Li, C.; Müllen, K. Toward Perylene Dyes by the Hundsdiecker Reaction. Org. Lett. 2014, 16, 2814-2817.

(53) Liu, M.; Liu, M.; She, L.; Zha, Z.; Pan, J.; Li, S.; Li, T.; He, Y.; Cai, Z.; Wang, J.; Zheng, Y.; Qiu, X.; Zhong, D. Graphene-Like Nanoribbons Periodically Embedded with Four- and EightMembered Rings. Nat. Commun. 2017, 8, 14924.

(54) Kolmer, M.; Zuzak, R.; Steiner, A. K.; Zajac, L.; Engelund, M.; Godlewski, S.; Szymonski, M.; Amsharov, K. Fluorine-programmed nanozipping to tailored nanographenes on rutile $\mathrm{TiO}_{2}$ surfaces. Science 2019, 363, 57-60.

(55) Kolmer, M.; Steiner, A. K.; Izydorczyk, I.; Ko, W.; Engelund, M.; Szymonski, M.; Li, A.-P.; Amsharov, K. Rational synthesis of atomically precise graphene nanoribbons directly on metal oxide surfaces. Science 2020, 369, 571-575.

(56) Batra, A.; Cvetko, D.; Kladnik, G.; Adak, O.; Cardoso, C.; Ferretti, A.; Prezzi, D.; Molinari, E.; Morgante, A.; Venkataraman, L. Probing the Mechanism for Graphene Nanoribbon Formation on Gold Surfaces Through X-Ray Spectroscopy. Chem. Sci. 2014, 5, 4419-4423.

(57) Gottardi, S. Designing Molecular Nano-Architectures on Metals and On Graphene. PhD Thesis, University of Groningen, 2015.

(58) Kimouche, A.; Ervasti, M. M.; Drost, R.; Halonen, S.; Harju, A.; Joensuu, P. K.; Sainio, J.; Liljeroth, P. Ultra-Narrow Metallic Armchair Graphene Nanoribbons. Nat. Commun. 2015, 6, 10177.

(59) Shi, K. J.; Yuan, D. W.; Wang, C. Z.; Shu, C. H.; Li, D. Y.; Shi, Z. L.; Wu, X. Y.; Liu, P. N. Ullmann Reaction of Aryl Chlorides On Various Surfaces and The Application in Stepwise Growth of 2D Covalent Organic Frameworks. Org. Lett. 2016, 18, 1282-1285.

(60) Panighel, M.; Quiroga, S.; Brandimarte, P.; Moreno, C.; GarciaLekue, A.; Vilas-Varela, M.; Rey, D.; Sauthier, G.; Ceballos, G.; Peña, D.; Mugarza, A. Stabilizing Edge Fluorination in Graphene Nanoribbons. ACS Nano 2020, 14, 11120-11129. 
(61) Bronner, C.; Björk, J.; Tegeder, P. Tracking and Removing Br during the On-Surface Synthesis of a Graphene Nanoribbon. J. Phys. Chem. C 2015, 119, 486-493.

(62) Tran, V.; Pham, T. A.; Grunst, M.; Kivala, M.; Stöhr, M. Surface-Confined [2+2] Cycloaddition Towards One-Dimensional Polymers Featuring Cyclobutadiene Units. Nanoscale 2017, 9, 18305.

(63) Abyazisani, M.; MacLeod, J. M.; Lipton-Duffin, J. Cleaning up after the Party: Removing the Byproducts of On-Surface Ullmann Coupling. ACS Nano 2019, 13, 9270-9278.

(64) Sánchez-Sánchez, C.; Martínez, J. I.; Ruiz del Arbol, N.; Ruffieux, P.; Fasel, R.; López, M. F.; De Andres, P. L.; Martín-Gago, J. A. On-Surface Hydrogen-Induced Covalent Coupling of Polycyclic Aromatic Hydrocarbons via a Superhydrogenated Intermediate. J. Am. Chem. Soc. 2019, 141, 3550-3557.

(65) Gross, L.; Mohn, F.; Moll, N.; Liljeroth, P.; Meyer, G. The Chemical Structure of a Molecule Resolved by Atomic Force Microscopy. Science 2009, 325, 1110-1114.

(66) Lawrence, J.; Brandimarte, P.; Berdonces-Layunta, A.; Mohammed, M. S. G.; Grewal, A.; Leon, C. C.; Sanchez-Portal, D.; de Oteyza, D. G. Probing the Magnetism of Topological End States in 5-Armchair Graphene Nanoribbons. ACS Nano 2020, 14, 44994508.

(67) Albrecht, F.; Pavlicek, N.; Herranz-Lancho, C.; Ruben, M.; Repp, J. Characterization of a Surface Reaction by Means of Atomic Force Microscopy. J. Am. Chem. Soc. 2015, 137, 7424-7428.

(68) van der Lit, J.; Boneschanscher, M. P.; Vanmaekelbergh, D.; Ijäs, M.; Uppstu, A.; Ervasti, M.; Harju, A.; Liljeroth, P.; Swart, I. Suppression of Electron-Vibron Coupling in Graphene Nanoribbons Contacted via a Single Atom. Nat. Commun. 2013, 4, 2023.

(69) Pavliček, N.; Mistry, A.; Majzik, Z.; Moll, N.; Meyer, G.; Fox, D. J.; Gross, L. Synthesis and Characterization of Triangulene. Nat. Nanotechnol. 2017, 12, 308-311.

(70) Di Giovannantonio, M.; Eimre, K.; Yakutovich, A. V.; Chen, Q.; Mishra, S.; Urgel, J. I.; Pignedoli, C. A.; Ruffieux, P.; Müllen, K.; Narita, A.; Fasel, R. On-Surface Synthesis of Antiaromatic and OpenShell Indeno[2,1-b]fluorene Polymers and Their Lateral Fusion into Porous Ribbons. J. Am. Chem. Soc. 2019, 141, 12346-12354.

(71) Kawai, S.; Sadeghi, A.; Okamoto, T.; Mitsui, C.; Pawlak, R.; Meier, T.; Takeya, J.; Goedecker, S.; Meyer, E. Organometallic Bonding in an Ullmann-Type On-Surface Chemical Reaction Studied by High-Resolution Atomic Force Microscopy. Small 2016, 12, 53035311.

(72) Soler, J. M.; Artacho, E.; Gale, J. D.; García, A.; Junquera, J.; Ordejón, P.; Sánchez-Portal, D. The SIESTA Method for Ab Initio Order-N Materials Simulation. J. Phys.: Condens. Matter 2002, 14, 2745-2779.

(73) Hapala, P.; Kichin, G.; Wagner, C.; Tautz, F. S.; Temirov, R.; Jelínek, P. Mechanism of High-Resolution STM/AFM Imaging with Functionalized Tips. Phys. Rev. B: Condens. Matter Mater. Phys. 2014, 90, 085421.

(74) Hapala, P.; Temirov, R.; Tautz, F. S.; Jelínek, P. Origin of HighResolution IETS-STM Images of Organic Molecules with Functionalized Tips. Phys. Rev. Lett. 2014, 113, 226101.

(75) Kittelmann, M.; Rahe, P.; Nimmrich, M.; Hauke, C. M.; Gourdon, A.; Kühnle, A. On-Surface Covalent Linking of Organic Building Blocks on a Bulk Insulator. ACS Nano 2011, 5, 8420-8425. 